
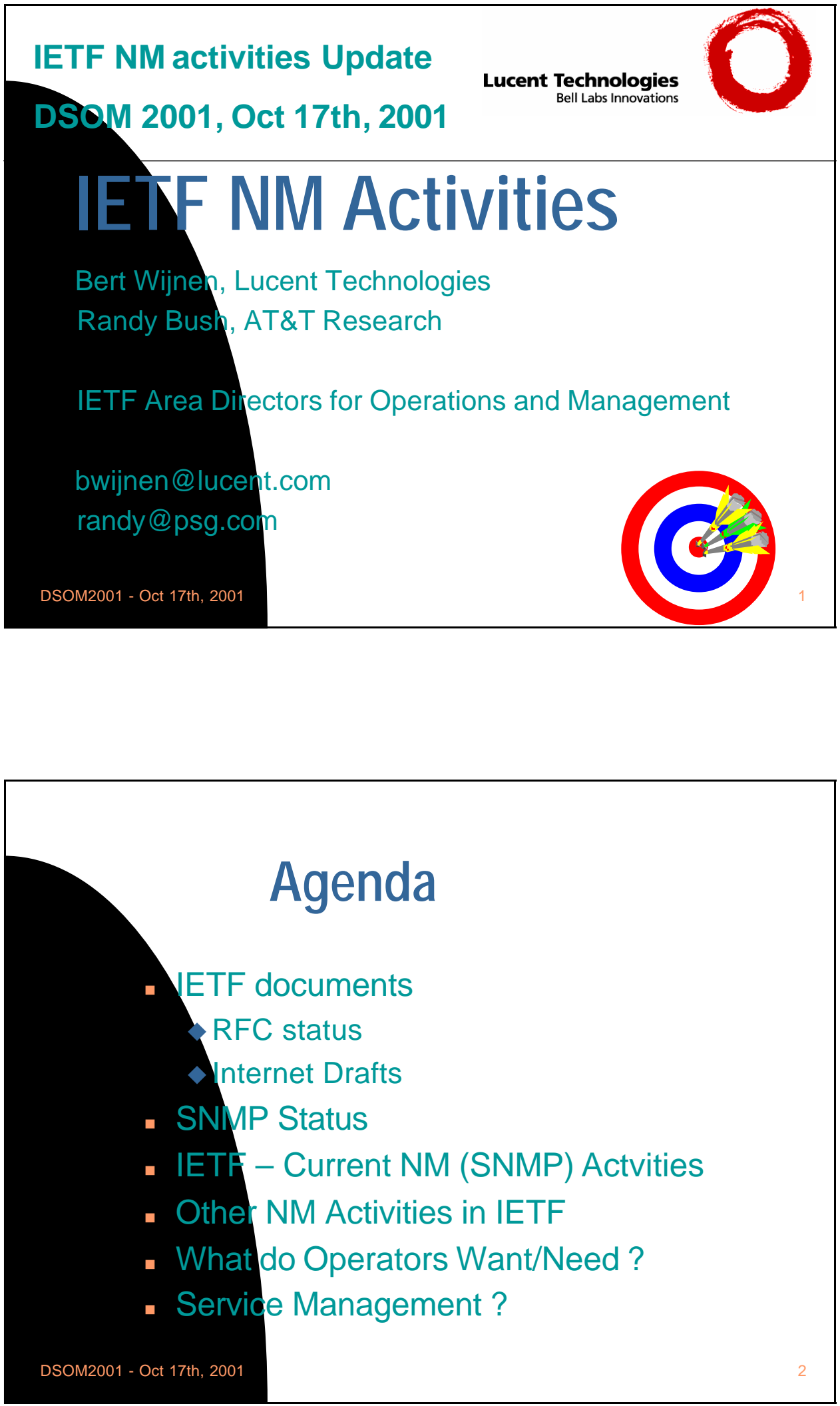

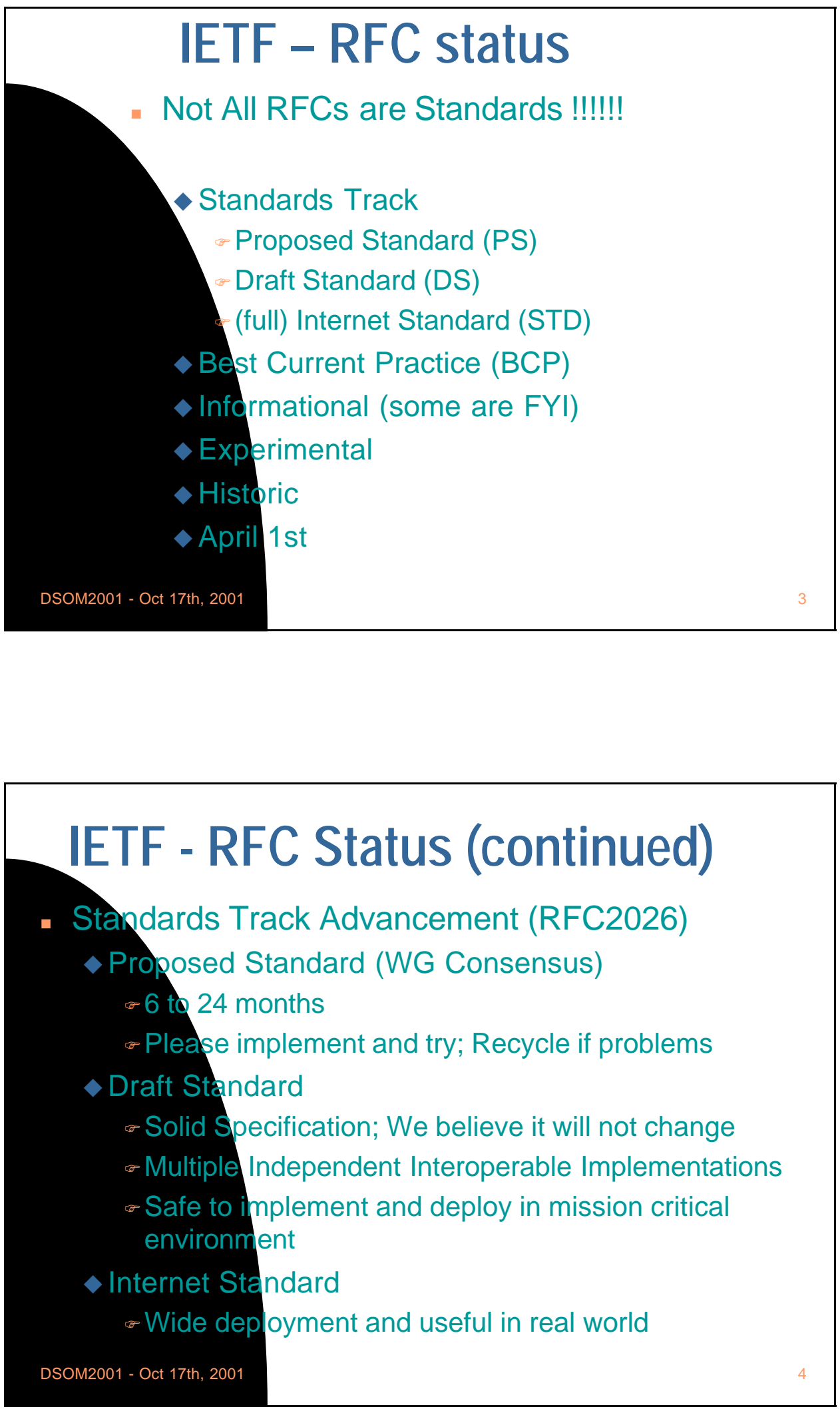


\section{Internet Drafts}

- Not all Internet Drafts are WG or IETF endorsed documents

- MostWG documents are named

- Dratt-ietf-<wg-name>-something-nn.txt

- Most non-WG documents are named

- Draft-ietf-<author>-something-nn.txt

$\rightarrow$ Draft-ielf-<author $>-<$ wg-name>-something-nn.txt

- See http://www. ietf.org/html.charters/wg-dir.html

- For all WG charters and WG documents

\section{SNMP Status - versions}

- SNMP Message Wrappers:

SNMPV1

$\diamond$ SNMPV2C

- SNMPV3

- SNMPProtocol Operations (PDUs):

- SNMPV1 Protocol Operations

- SNMPV2 Protocol Operations

- Structure of Management Information (SMI):

• SMlv1

$\diamond$ SMIv2 


\section{SNMP Status - version 1}

SNMPv1 message wrapper

no Security,

A.e. community string (plain text password)

- SNMPv1 Protocol Operations

$\diamond$ GET, GETNEXT,

$\triangle$ SET

$\diamond$ GETRESPONSE,

$\checkmark$ TRAPV1

- SMlv1 data types

$\diamond$ MIBs in SMlv1 format

\section{SNMP Status - version 1 (continued)}

SNMPv1 - (Full) Internet Standard

\section{RFC1157 (STD 15)}

Specifies Message Wrapper

Specifies Protocol Operations (PDUs)

- SMlv1 - (Full) Internet Standard

$\checkmark$ RFC155 and RFC1212 (STD16)

$\checkmark$ RFC1215 (informational)

- MIB II(Full) Internet Standard

$\diamond \mathrm{RFC} 1213$ (STD 17)

- Various Other MIBs (Proposed and Draft Stds) 

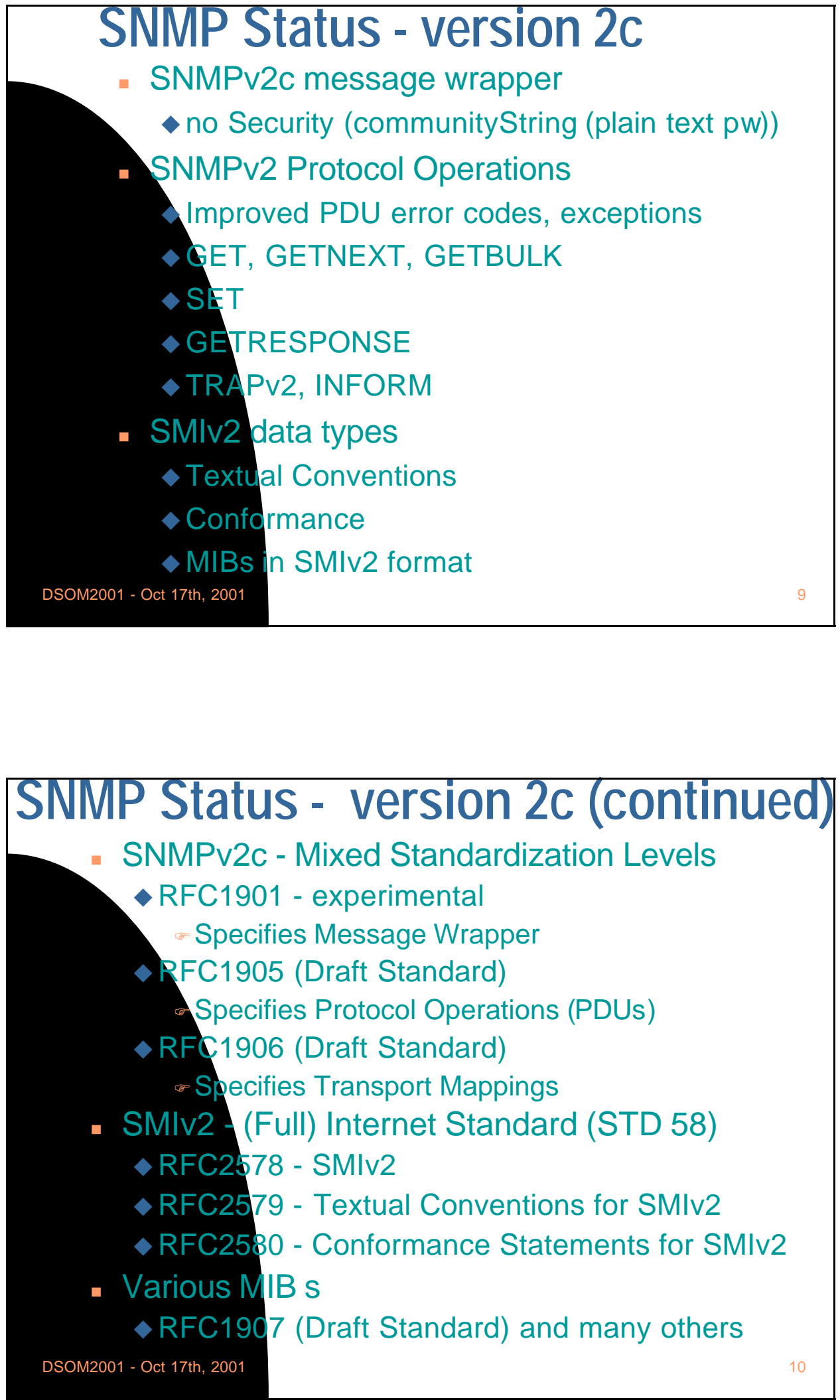

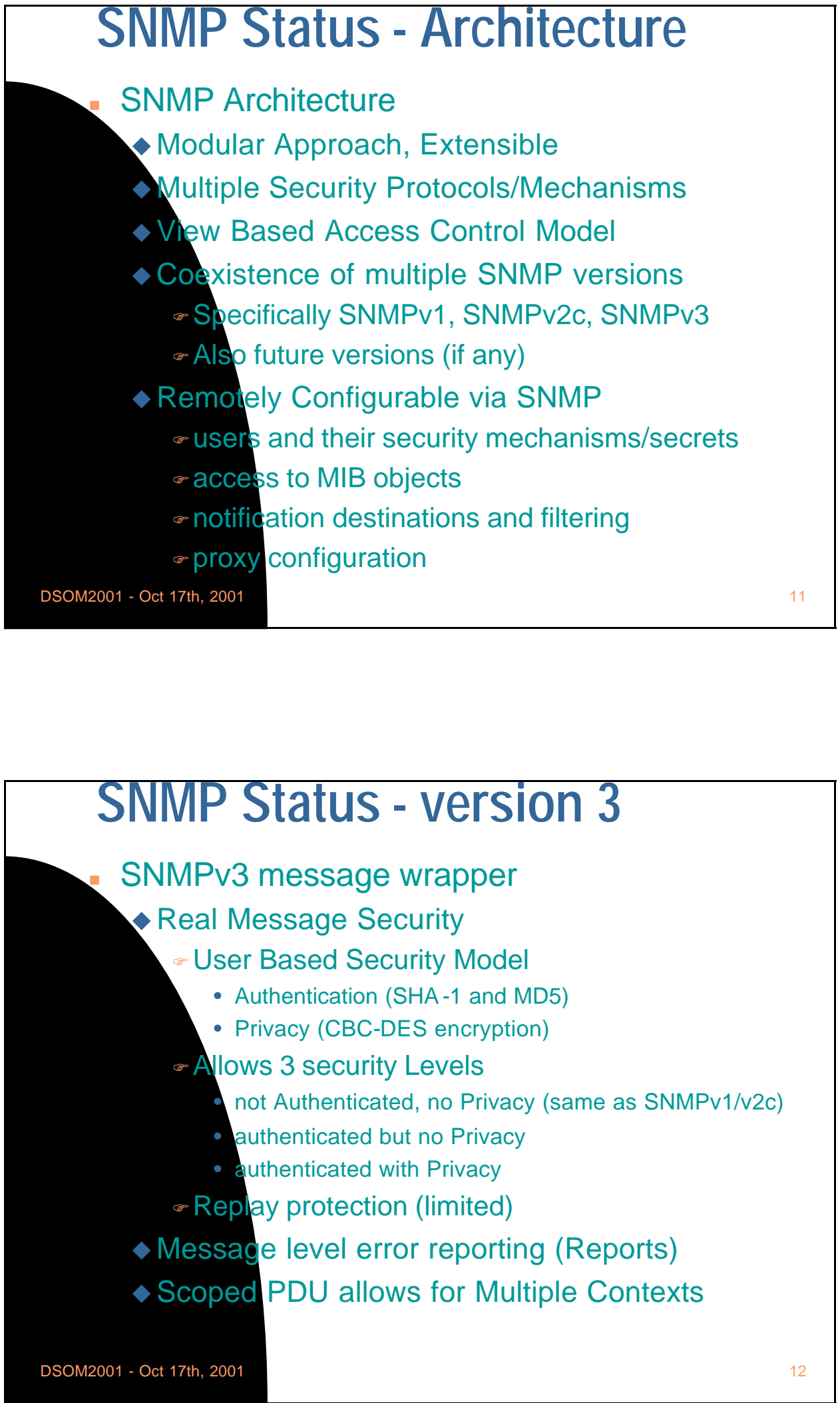


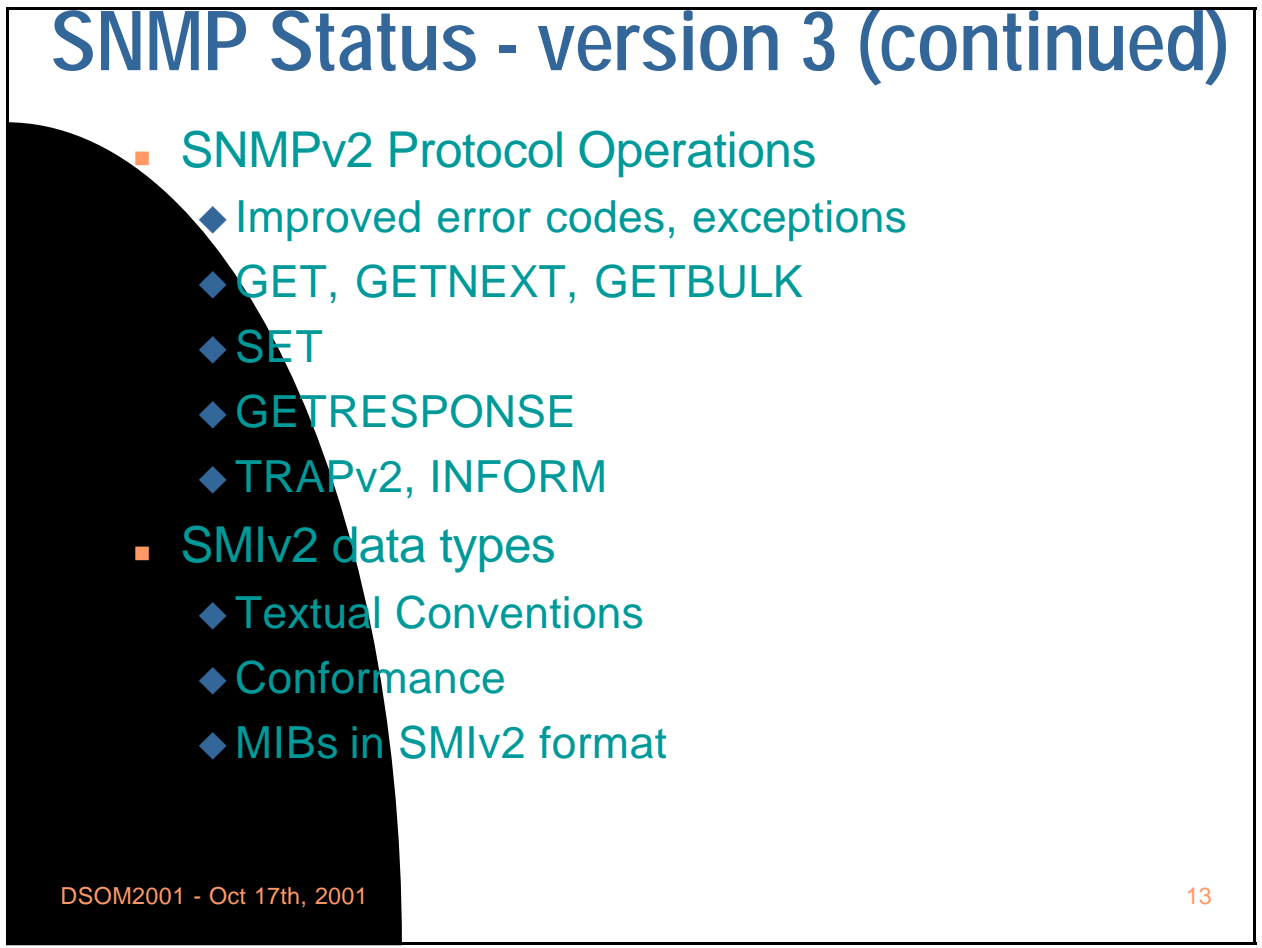

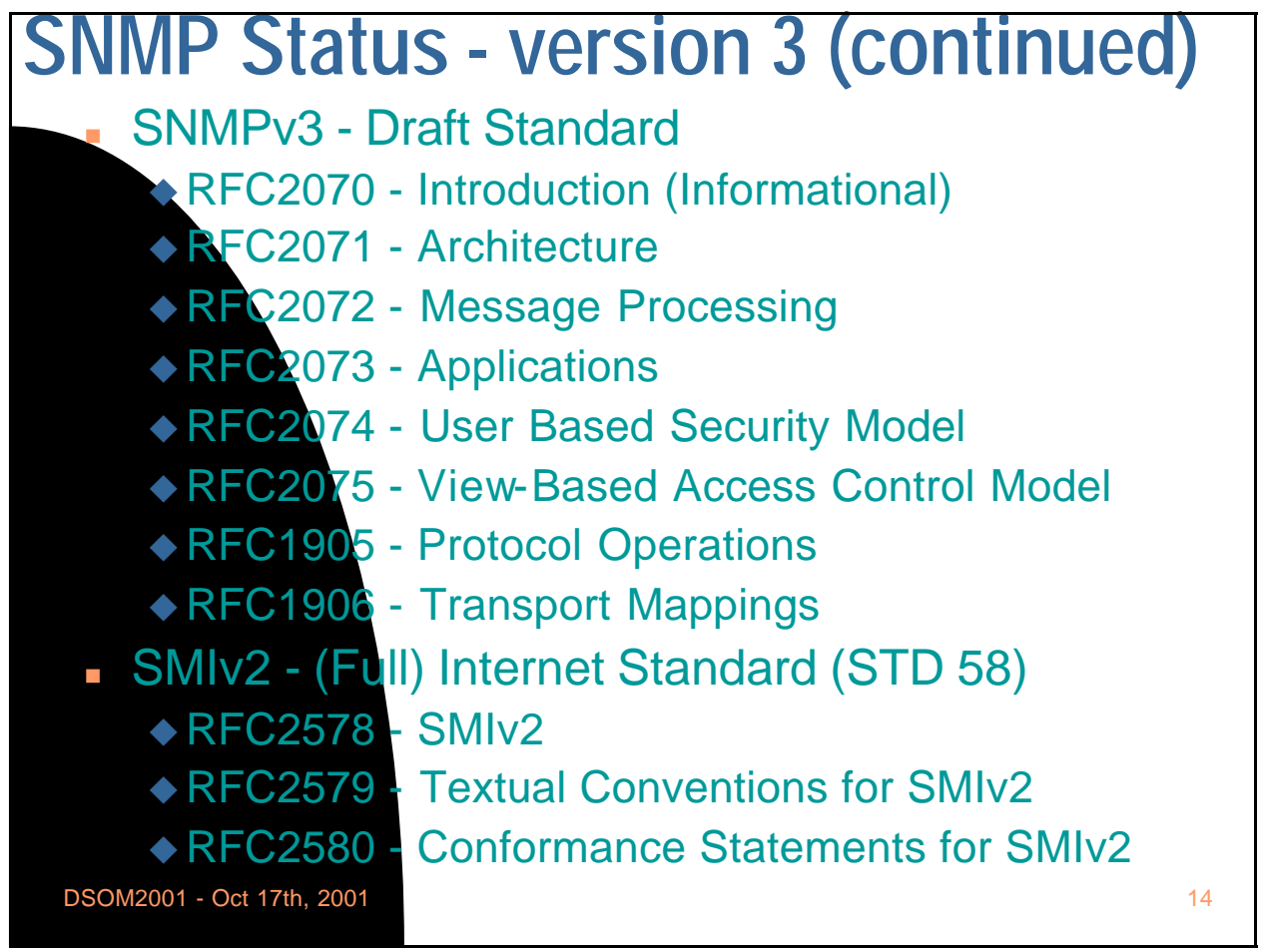




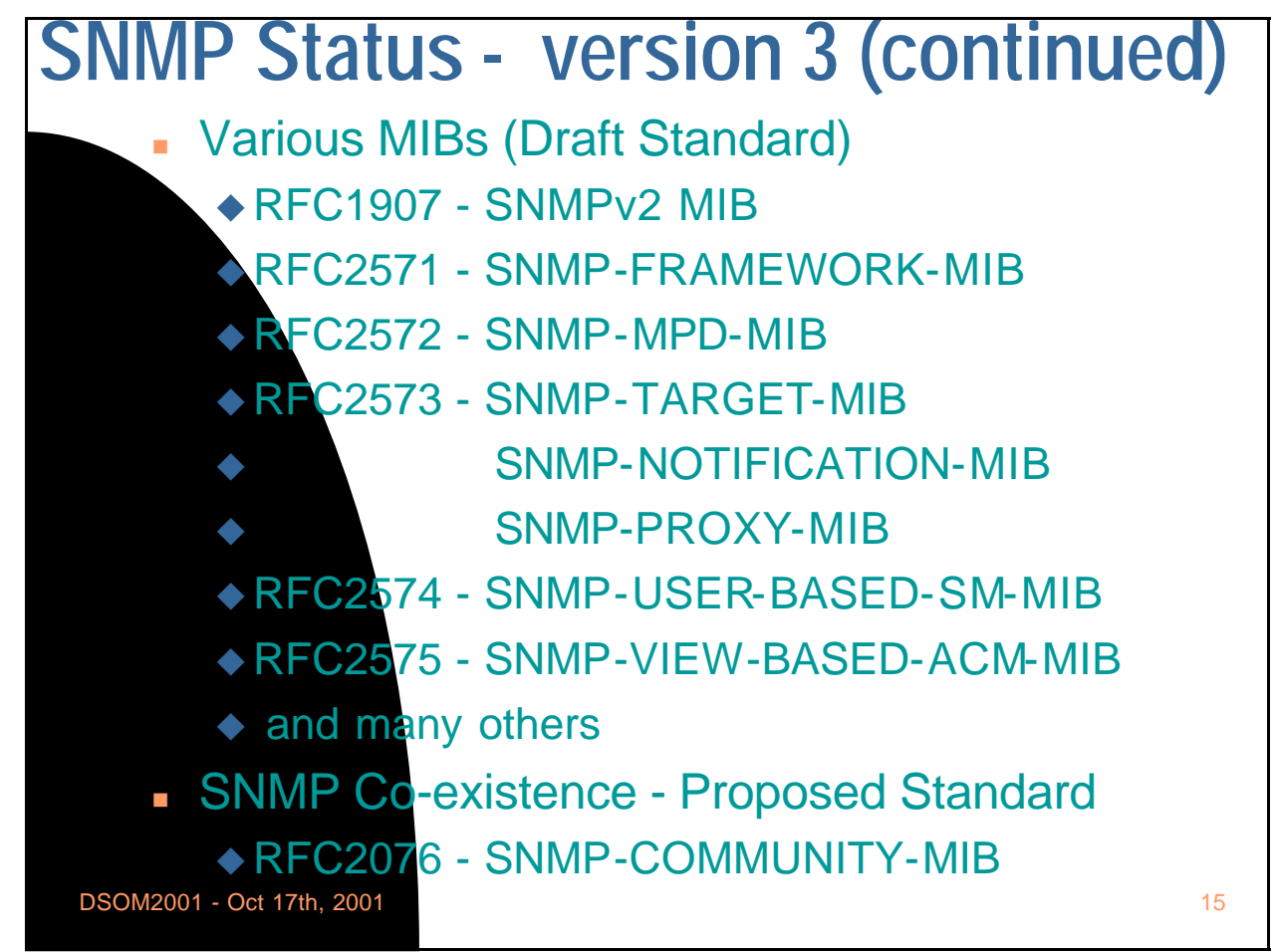

\section{SNMP Status - SNMPv3 Features}

- Comes with Modular and Extensible Architecture
- BetBulk, Inform
- Security and Access Control to MIB objects
- Remote Configuration of SNMP Engine
- Coexistence with SNMPv1 and SNMPv2c
- Over 10 interoperable implementations
- Various vendors are shipping
- Deployment reports coming in
- See http://www.ibr.cs.tu-bs.de/projects/snmpv3/
Dsom2001 - oct 17th, 2001




\section{IETF - Current NM Activities 1/5}

- Ongoing work SNMPv3 WG

- advance SNMPv3 to Full Internet Standard

- SNMPCONF WG

- Configuration Management with SNMP

- Policy-Based Network-Wide Configuration Management

- SMlng WG-Next Generation of SMI

- More Object Oriented

- Merge SPPI and SMI

- EOS WG - Evolution Of SNMP

$\checkmark$ Performance Enhancements

- Within SNMP Architecture (RFC2571)

\section{IETF - Current NM Activities 2/5}

- DISMAN WG - Distributed Management

$\checkmark$ Adrance various MIBs to Draft Standard

- Remote Operations (Ping, Traceroute, dnslookup)

- Script and Scheduling MIBs

- Expression, Event and Notification Logging MIBs

$\rightarrow$ New ALARM MIB

- RMONMIB WG - Remote Monitoring

- Advance RMON1 and RMON2 MIBs to Full Standard

- Including high capacity versions

$\checkmark$ APM MIB (Application Performance Monitoring)

$\checkmark$ DSMON MIB (Diffserv Monitoring)

$\diamond$ etc

DSOM2001 - Oct 17th, 2001 

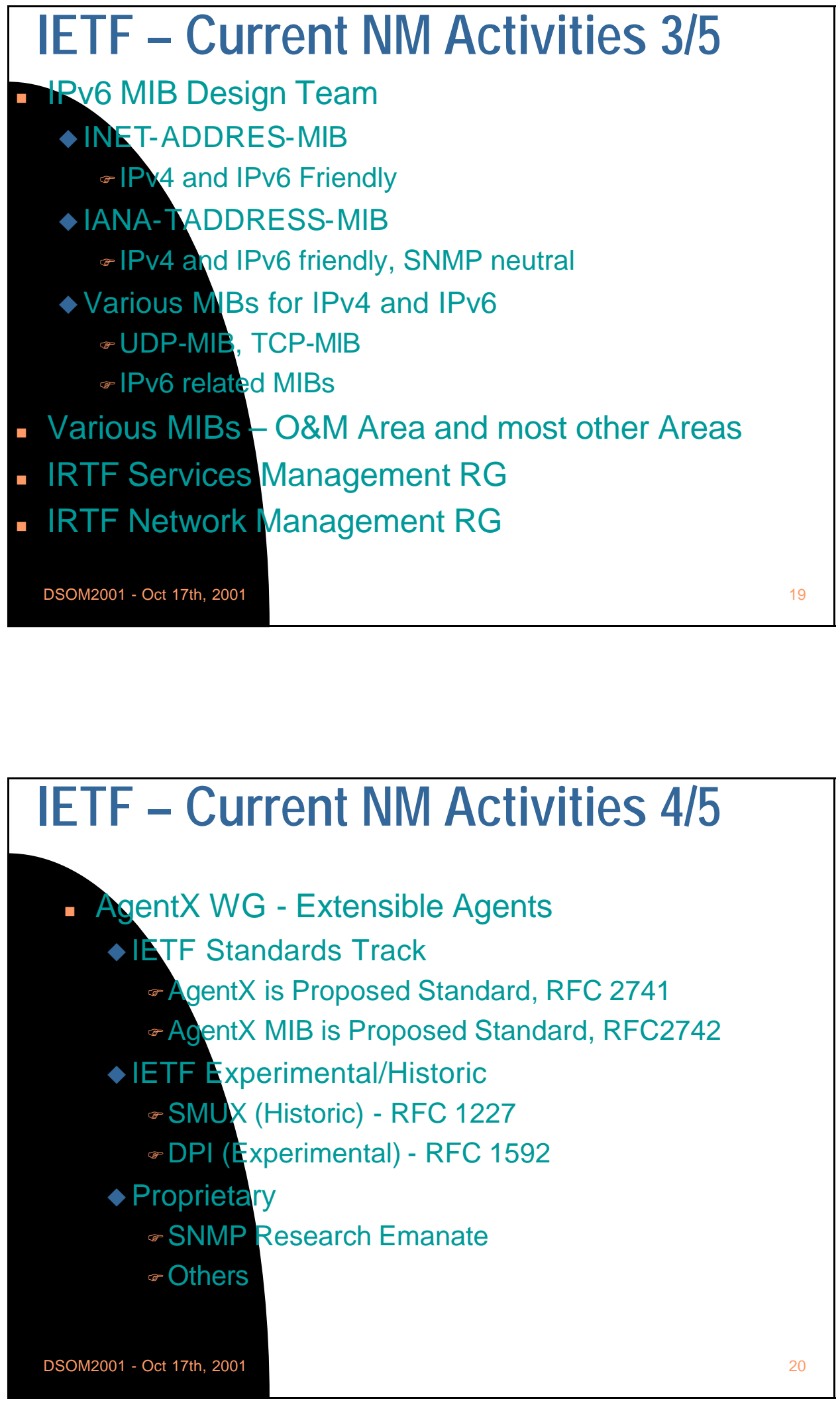

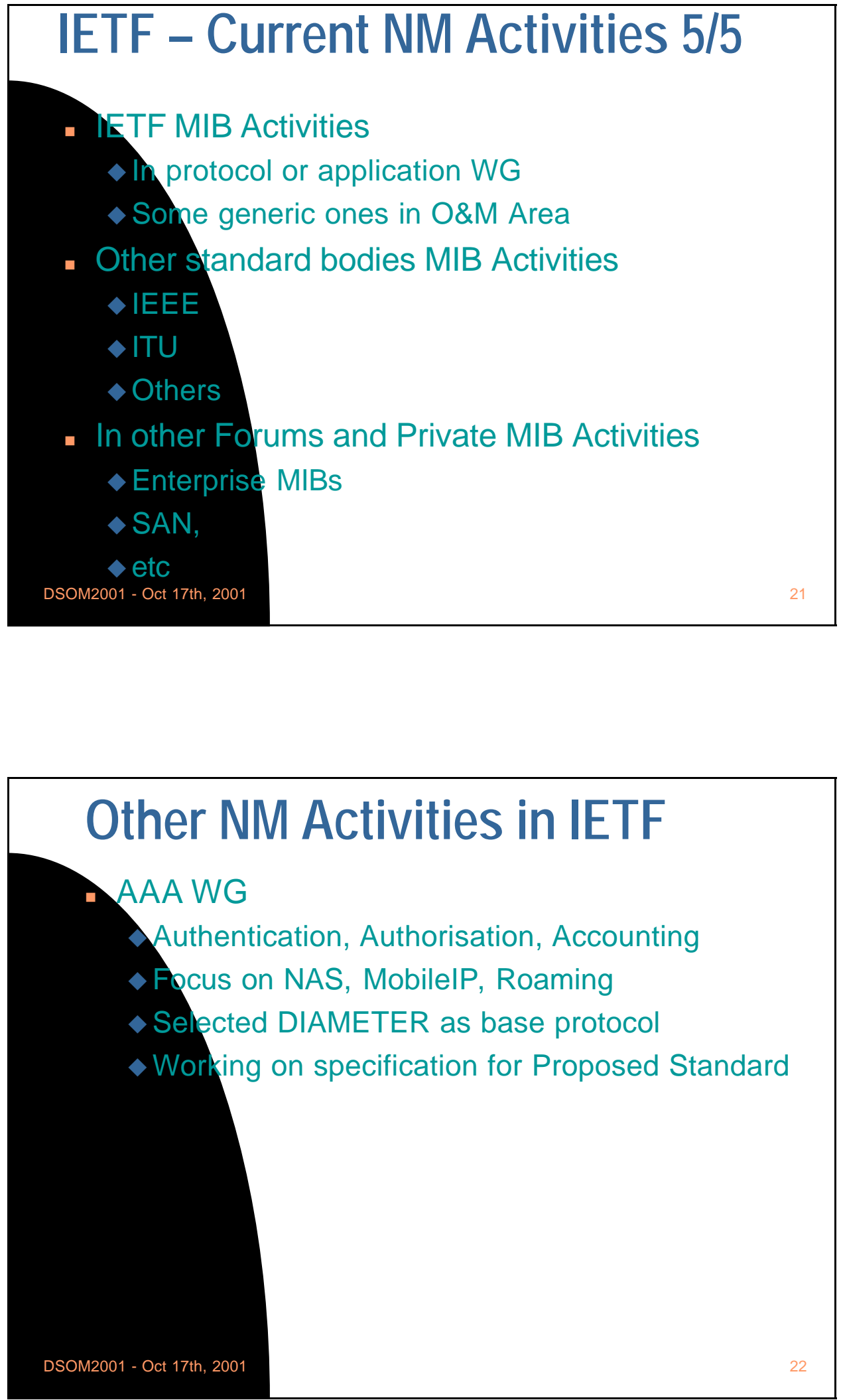


\section{Other NM Activities in IETF}

Policy Framework WG
Policy Core Information Model
Proposed Standard (RFC3060)
× Policy Core Information Model Extensions
> Policy Framework (??)
× Policy QoS Information Model
× Policy Device Information Model
> Policy Terminology (approved as RFC)

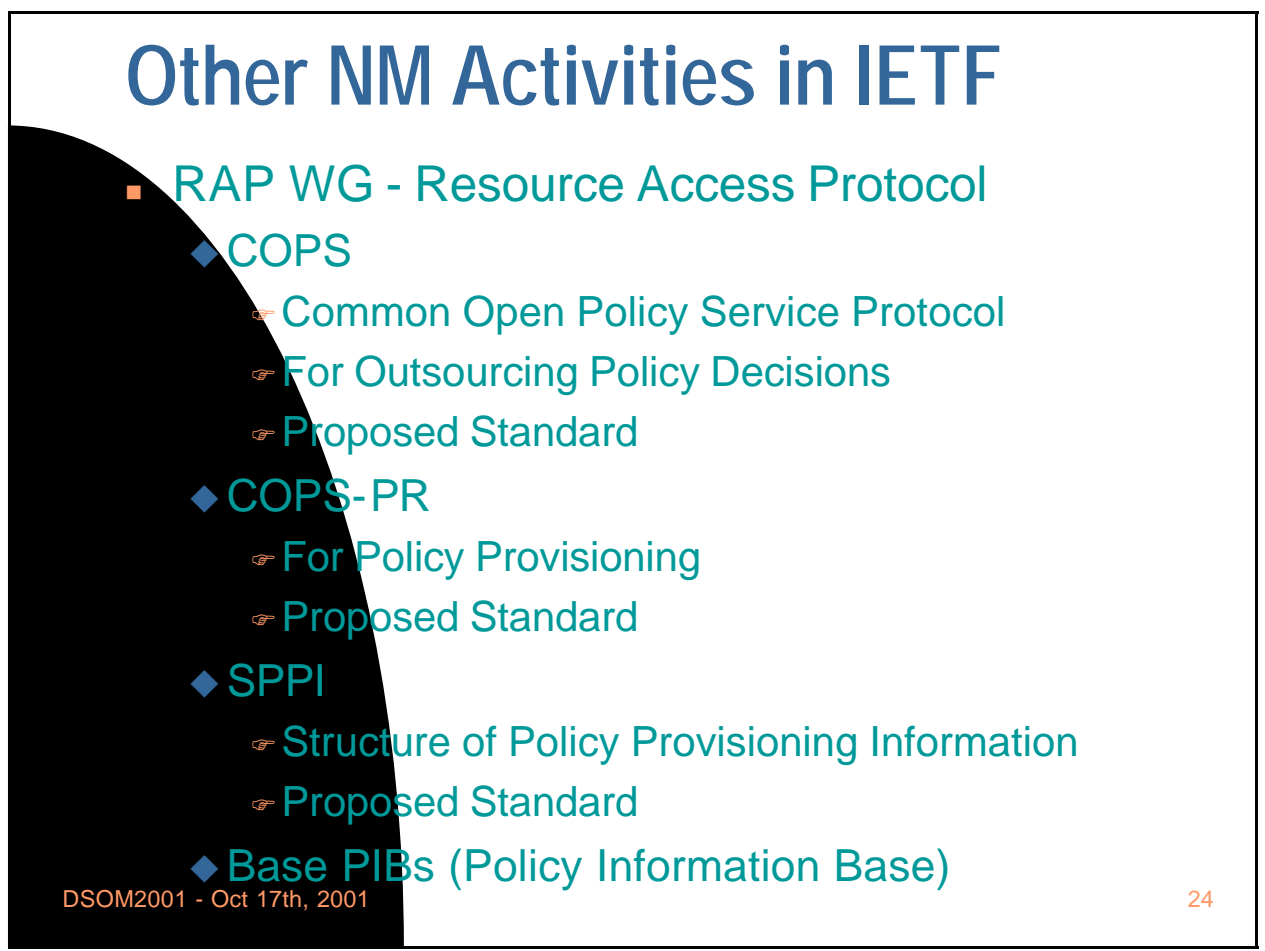



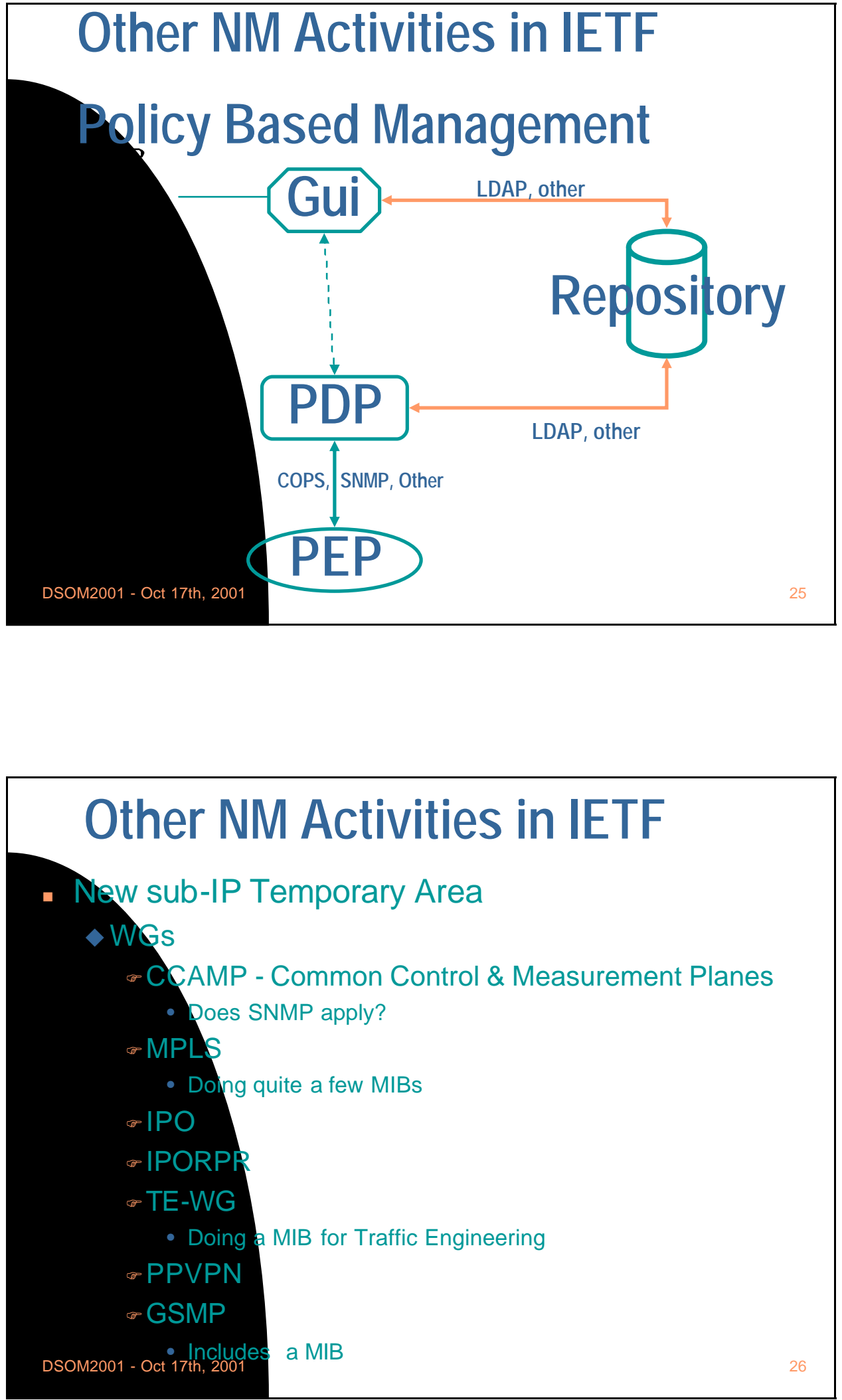


\section{What do Users/Operators Want?}

- Plain CLI

- Text based interfaces aka SMTP

- See

- Draft-ops-operator-req-mgmt-00.txt

- We had (will have) meetings with operators

$\checkmark$ At IETF 50 and 51

$\checkmark$ At NANOG (core IP network ISPs)

$\diamond$ At RIPE (IP ISPs)

- At LISA (in December 2001, Enterprise networks?)

- Not much talk about service management

\section{Service Management?}

- A look at TMN

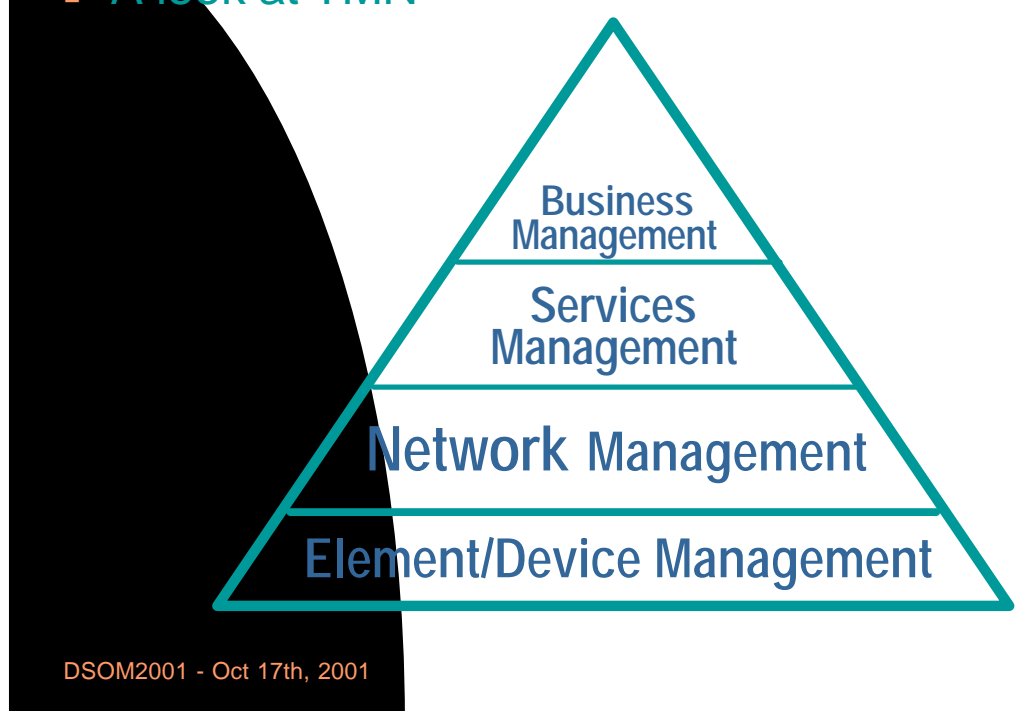




\section{Service Management?}

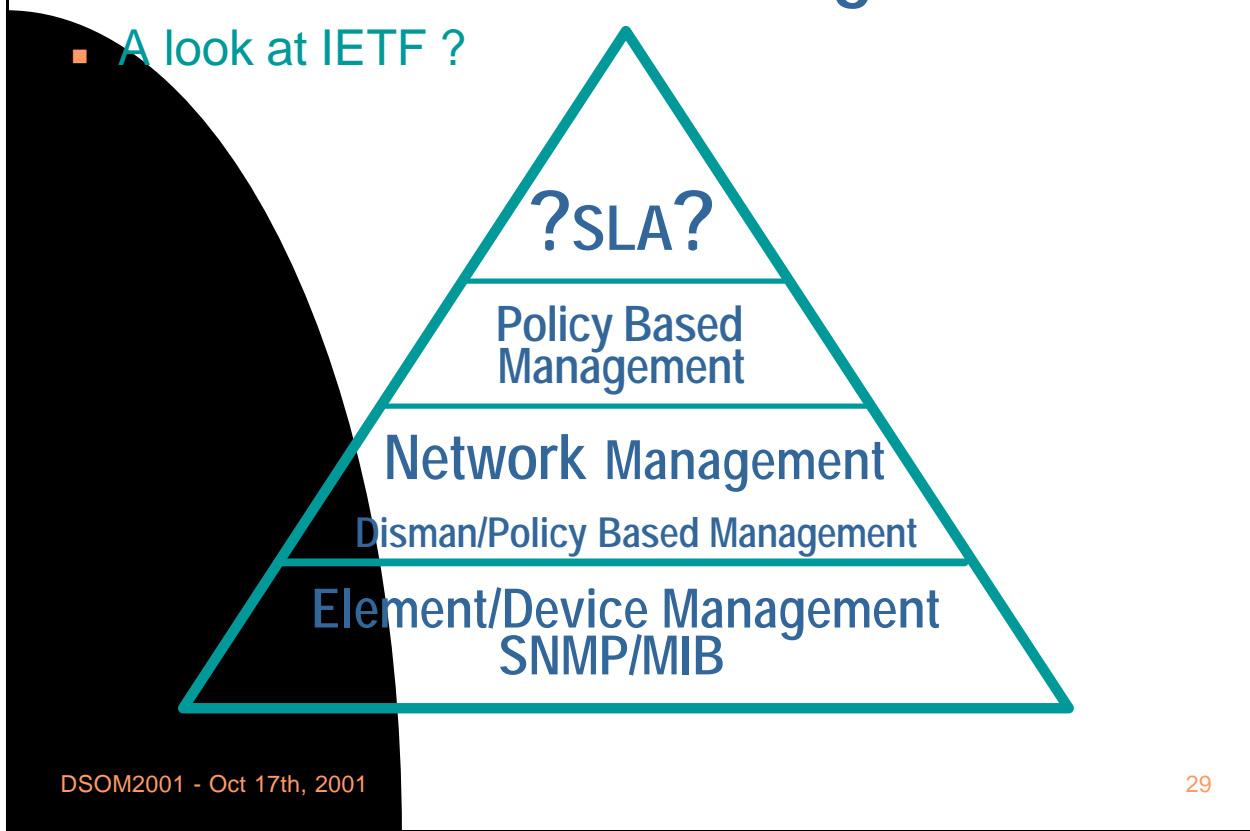

\section{Service Management?}

Do Vendors want it Standardized?

In fact do they want NM Protocol standards?

Do they want standard MIBs

$\checkmark$ Is there an incentive? Only for Startups?

$\checkmark$ Operators want std protocol and MIBs

- Allows them to mix and match

- Do operators want Standardized SM ?

- I am not sure

- It may take away their competetive advantage

- If they do... they should step up to the task

- IRTF Services Management RG 


\section{More Information}

- http://www.ietf.org/html.charters/wg-dir.html

$\diamond$ Forall current IETF Working Groups

- http://www.ietf.org

$>$ Starting point for IETF information

- http://wwmops.org

^ Starting point for OPS Area specific web pages

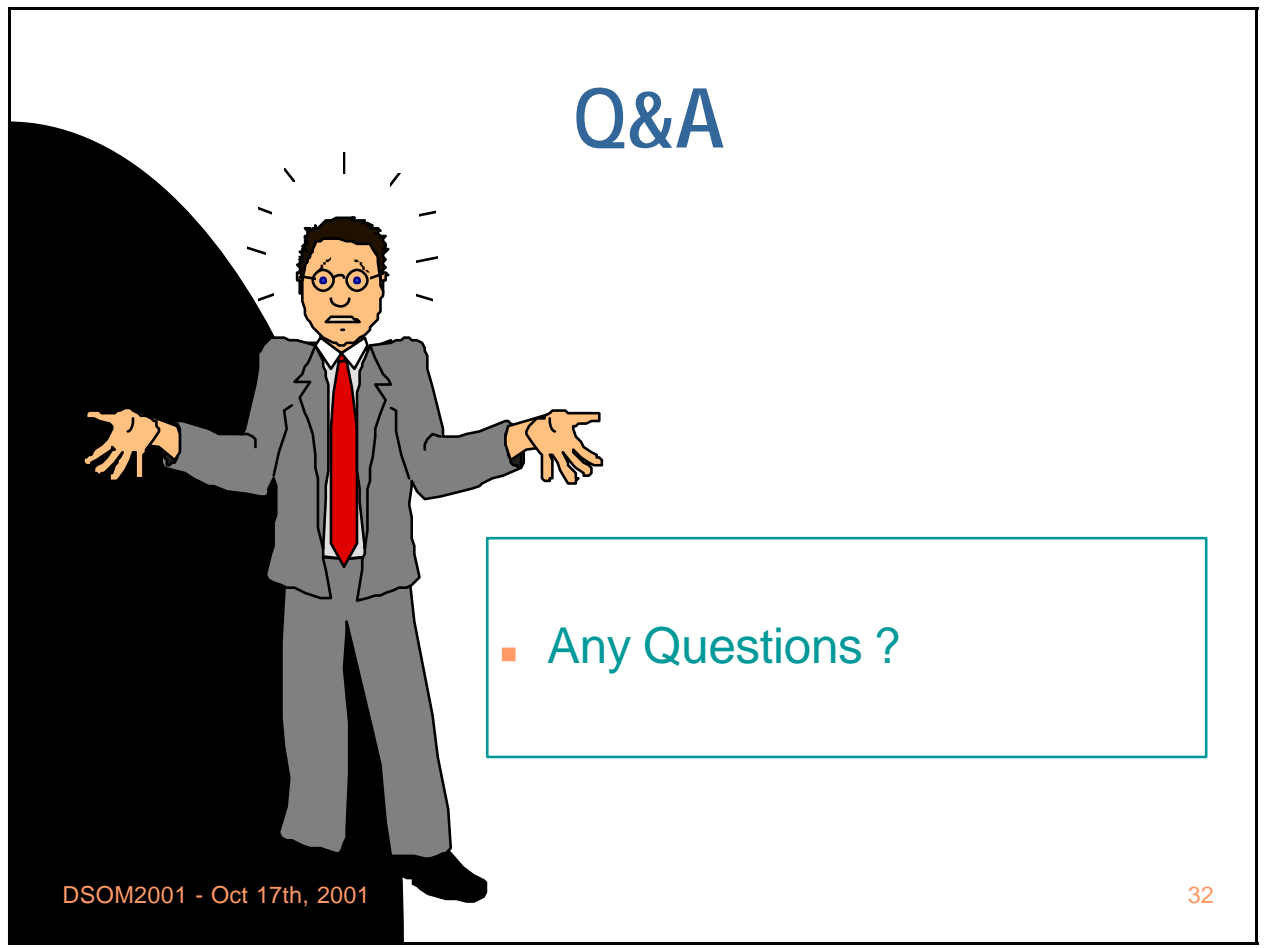

